\title{
EM Characterization of Self Hardening CrAIN/BN Nanocomposite Coatings
}

\author{
Masateru Nose ${ }^{*}$, Wen-An Chiou ${ }^{* *}$, Li-Chung Lai ${ }^{* *}$, Tomohiro Watanuki*** and Kenji Matsuda ${ }^{* * *}$ \\ *Faculty of Art and Design, University of Toyama, 180 Futagami-machi, Takaoka 933-8588, Japan \\ ** NISP Laboratory, NanoCenter, University of Maryland, College Park, MD 20740-2831 \\ ***School of Science and Engineering, University of Toyama, 3190 Gofuku 930-8555, Japan
}

It is well known that some nanocomposite coatings such as TiBN and TiCrBN display a "self hardening phenomena" after annealing [1]. Furthermore, this self hardening phenomenon is observed only in non-oxidizing environments such as vacuum or inert gas atmosphere. To date, little is known about the self hardening phenomena in quaternary CrAlBN systems; however, recent work has shown that some CrAIN/BN nanocomposite coatings exhibit peculiar oxidation resistance, i.e., after annealing of these coatings in ambient air in the range of 700 to $800^{\circ} \mathrm{C}$, the indentation/plastic hardness increased in the order of 8 to $30 \%$ [2, and Fig. 1]. This peculiar phenomenon of the CrAlN/BN coatings was investigated by examining the microstructure and microchemistry of the coating heated in different environments.

$\mathrm{Cr}_{50} \mathrm{Al}_{50}$ alloy $(99.8 \%)$ and $h$-BN (99.5\%) targets were sputtered in a mixture of highly purified argon and nitrogen gases (99.9999\%). The working pressure was kept at $\sim 0.25 \mathrm{~Pa}$ ( 2 mTorr). The pulsed dc input power (frequency, $240 \mathrm{kHz}$; duty cycle, 75-78\%) and rf power (13.56 MHz) were supplied to $\mathrm{Cr}_{50} \mathrm{Al}_{50}$ alloy and $h$-BN targets, respectively. The high speed steel (HSS) substrates were preheated to about $350{ }^{\circ} \mathrm{C}$ at the beginning of the deposition and rotated at $10 \mathrm{rpm}$ while CrAIN and BN were co-sputtered on the substrates. Total thickness of the film was in the range of $1.8 \sim 2.2 \mu \mathrm{m}$. Annealing of the sample coupons was carried out using a temperaturecontrolled tubular furnace in air, nitrogen and argon, respectively. Sample coupons were heated for exactly 1 hour and then cooled outside the furnace. Coating morphology, microstructure and microchemistry were examined with a FEG SEM (Hitachi-SU70) and a FEG TEM (JEOL-2100F) that equipped with Bruker and Oxford EDS system, respectively.

Figure 1 illustrates the change of plastic hardness, $H_{p l}$, of the $\mathrm{CrAlN} / 18 \mathrm{vol} \% \mathrm{BN}$ coatings at annealing temperature in different gas environments - air, nitrogen and argon atmospheres. Selfhardening of the coating was evidenced by the increases in plastic hardness that occurred after annealing to $800{ }^{\circ} \mathrm{C}$ in air. Using high resolution SEM, distinct morphological change in the form of miniscule bumps ( $30 \mathrm{~nm}$ in diameter) was observed only in samples annealed in air (Fig. 2). In low magnification cross-sectional TEM images, both as-deposited and annealed samples appeared similar with the CrAlN/18vol\%BN coating assuming a columnar structure (Figs. 3a and 4a); however, higher magnification TEM micrographs show a disruption in the columnar structure by a very thin layer $(20 \sim 40 \mathrm{~nm})$ of film in the annealed sample that is not observed in the as-deposited sample (Figs 3b and 4b). The HRTEM image (Fig. 4b) reveals that the top most layer is characterized by amorphous materials with embedded nanocrystalline particles (white circles in Fig. 4b). EDS line profiles of cross-sectional samples depict a high concentration of $\mathrm{O}$ in the upper most layer of annealed sample, while $O$ content remains constant throughout the columnar layer in the as-deposited sample (Figs. 3c and 4c). SEM EDS and EPMA WDS results also show the same elemental distribution pattern. The thin amorphous and nancrystallite-embedded layer found in the microstructure that was formed by oxidizing of the surface coating is likely to be one of the factors that responsible for the "self hardening phenomena" in CrAlN/BN coatings; further investigation of other factors contributing to this phenomenon is in progress.

[1] P. H. Mayhofer, et. al., Progress in Materials Science, 51 (2006) 1032-1114.

[2] M. Nose, et. al., Surf. \& Coat. Technol., in press

[3] This study was partially supported by NSF-MRSEC (DMR 05-20471) and NanoCenter at UMD. 

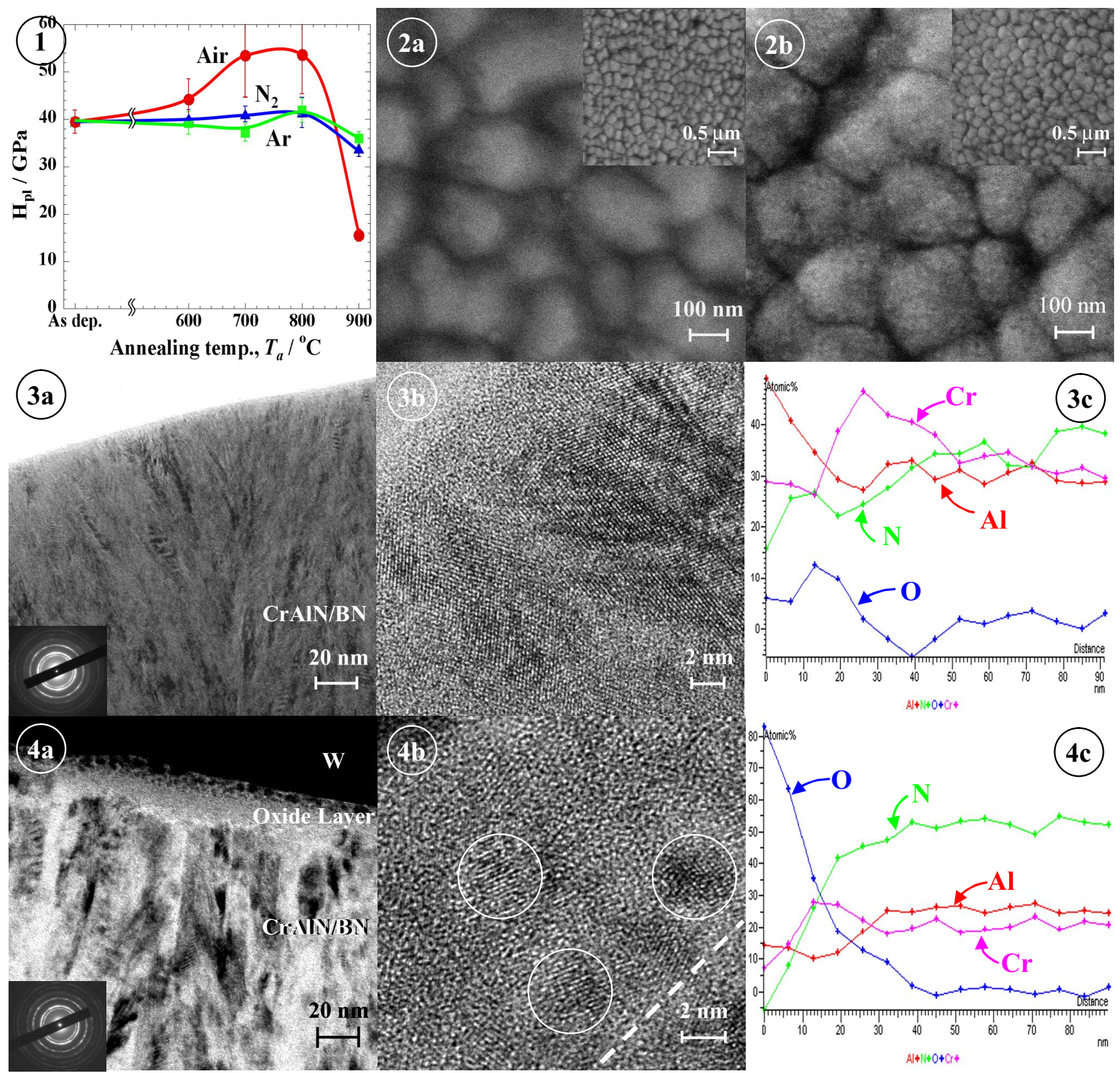

Fig. 1. Diagram shows the variation of plastic hardness, $\mathrm{H}_{\mathrm{pl}}$, of $\mathrm{CrAlN} / 18 \mathrm{vol} \% \mathrm{BN}$ thin film at different annealing temperatures in air, nitrogen and argon atmosphere, respectively.

Fig. 2. (a) HRSEM plane-view image of as-deposited film depicting a smooth film surface.

(b) HRSEM plane-view image showing a bumpy film surface after annealed at $800{ }^{\circ} \mathrm{C}$ in air.

Fig. 3. (a) Cross-sectional TEM image and SAD pattern of as-deposited CrAIN/18vol\%BN coating revealing fine columnar microstructure of the film.

(b) Cross-sectional HRTEM view of the surface of coating showing a columnar structure extended to the top of the film homogenously.

(c) EDS line profile depicting elemental concentration across the film.

Fig. 4. (a) Cross-sectional TEM image of after annealing $\left(800{ }^{\circ} \mathrm{C}\right.$ for $1 \mathrm{hr}$.) in air showing an oxide layer formed on the surface of CrAlN $/ 18 \mathrm{vol} \% \mathrm{BN}$ thin film and also column grain growth.

(b) HRTEM image of the oxide layer with nanocrystallites embedded in the amorphous layer.

(c) EDS line profile showing high oxygen concentration in the upper most oxidized layer. 